\title{
Testing Van Gool's Hypothesis: A Method to Predict Side Effects of Cholinesterase Inhibitors in Patients with Cellular Degenerative and Vascular Dementia
}

\author{
Liza van Eijk, ${ }^{1}$ Erwin R. Groot, ${ }^{2}$ Maritza van Spronsen, ${ }^{3}$ Yvonne J. Maas, ${ }^{3}$ Daniel Keeser, ${ }^{4}$ \\ Oliver Pogarell, ${ }^{4}$ and Hessel J. Engelbregt ${ }^{4,5}$
}

\author{
${ }^{1}$ Department of Clinical Neuropsychology, VU University, 1081 BT Amsterdam, Netherlands \\ ${ }^{2}$ Department of Geriatrics, Albert Schweitzer Hospital, 3318 AT Dordrecht, Netherlands \\ ${ }^{3}$ Faculty of Social Sciences, Leiden University, 2300 RB Leiden, Netherlands \\ ${ }^{4}$ Department of Psychiatry and Psychotherapy, Ludwig-Maximilian University, 80539 Munich, Germany \\ ${ }^{5}$ Hersencentrum, 1016 XW Amsterdam, Netherlands
}

Correspondence should be addressed to Liza van Eijk; liza@hersencentrum.nl

Received 9 November 2014; Revised 10 May 2015; Accepted 25 May 2015

Academic Editor: Stavros Baloyannis

Copyright (C) 2015 Liza van Eijk et al. This is an open access article distributed under the Creative Commons Attribution License, which permits unrestricted use, distribution, and reproduction in any medium, provided the original work is properly cited.

\begin{abstract}
This study investigates a method to predict medical outcome of cholinesterase inhibitors in patients with Alzheimer's disease (AD) and vascular dementia (VaD). Van Gool predicts that patients with cholinergic deficit symptoms will benefit from treatment whereas patients without will experience side effects because of overstimulation of the cholinergic system. We predicted that $\mathrm{AD}$ and $\mathrm{VaD}$ patients with a longer RT experience fewer side effects than patients with a faster response and that VaD patients have a longer RT than $\mathrm{AD}$ patients. A number of 71 patients with $\mathrm{AD}$ or $\mathrm{VaD}$ diagnosis were included. A sustained attention task was administered, as well as the MMSE and a questionnaire about side effects. Results indicated that VaD patients with a longer RT reported fewer side effects. Furthermore, patients with VaD had a longer RT than patients with AD. MMSE was negatively correlated with RT in the $\mathrm{VaD}$ group. Thus, the performance on the attention task seems associated with suffering from side effects and thus tends to predict medical outcome in $\mathrm{VaD}$, but not in $\mathrm{AD}$. Perhaps this attention task was not sensitive enough to measure cholinergic deficit symptoms in AD patients. Furthermore, different doses of medication might confound the effect for the AD group.
\end{abstract}

\section{Introduction}

According to the National Health Service (NHS) in the United Kingdom one in three elderly people will develop dementia, of which two thirds are women. The total number is rising as people tend to live longer. The most common types of dementia are Alzheimer's disease (AD) and vascular dementia (VaD). The Diagnostic and Statistical Manual of Mental Disorders (DSM) IV-TR characterizes both AD and $\mathrm{VaD}$ as multiple cognitive deficits manifested by memory impairment and one (or more) cognitive disturbances, like aphasia, apraxia, agnosia, or disturbances in executive functioning [1]. In AD these cognitive deficits should each cause significant impairment in social/occupational functioning and show a decline compared to previous functioning levels which should include a gradual onset and continuing decline. The addition for $\mathrm{VaD}$ is that there should be evidence of focal neurological signs and symptoms or neuroimaging evidence indicating cerebrovascular diseases that are judged to be etiologically related to the disturbance.

There is evidence for signs of degeneration of neurons in the cholinergic system in $\mathrm{VaD}$ and $\mathrm{AD}[2,3], 40 \%$ loss of cholinergic neurons in $\mathrm{VaD}$ and even $70 \%$ in $\mathrm{AD}$ cases [4]. Deficits in the cholinergic system are not exclusively restricted to dementia but are also known in other disorders like Lewybodies dementia, Parkinson's disease, and Down's syndrome [5]. Deficits in the cholinergic system are characterized by symptoms like lack of attention, problems 
with concentration, and decreased ability to detect and select relevant stimuli leading to restlessness, anxiety, and confusion [5]. Because of decreased connection with the outside world, delusions and hallucinations often occur. Therefore, performance on cognitive tests is usually abnormal although there is no focal cortical disorder present [5].

At this moment, dementia cannot be cured. However, medication is given to delay cognitive deterioration, which might postpone moving patients into nursing homes [6]. Cholinesterase inhibitors (ChEIs) are commonly prescribed to suppress the symptoms as a decrease in cholinergic neurotransmission might lead to the cognitive and behavioural disturbances in dementia, particularly in AD. This treatment can cause severe side effects, like gastrointestinal problems, insomnia, anorexia, nausea, diarrhea, vomiting $[7,8]$, alteration in the cardiovascular system [7, 9], and an increased risk of death (through cardiovascular problems) [7, 8]. This raises the question if there is a method to predict the efficacy of medication and its side effects.

Theoretical Framework. The hypothesis of Van Gool [5] suggests that in particular dementia patients who show cholinergic deficit symptoms will benefit from ChEIs whereas dementia patients without these symptoms will suffer from severe side effects because of overstimulation of the cholinergic system. Patients with cholinergic deficit symptoms have attention problems and thus will have a longer reaction time (RT). The following three hypotheses will be tested. (1) AD patients with a longer RT on the attention task will benefit more from treatment than patients with an average or faster RT and will therefore experience fewer side effects. (2) VaD patients with a longer RT on the attention task will benefit more from treatment than patients with an average or faster RT (and should experience less side effects). (3) As the task measures sustained attention, which is impaired at an early stage of $\mathrm{VaD}$ but usually preserved in $\mathrm{AD}[10,11]$, it is predicted that $\mathrm{VaD}$ patients have a longer RT on the attention task than $\mathrm{AD}$ patients. It is possible that cholinergic deficits are an extra factor in a longer RT; however, the main reason that RT is longer in vascular dementia than in Alzheimer's disease in general is because of other reasons. White matter and periventricular hyperintensities are most severe in vascular dementia versus other dementia types such as Alzheimer's disease [12]. Such abnormalities are related to deficits in cognition, like slowing of mental processing speed, decreased working memory, and impairment of abstract reasoning [13].

\section{Method}

This study included 421 patients with the diagnosis $\mathrm{AD}(n=$ 325 ; males $=151$; females $=174)$ or $\operatorname{VaD}(n=96$; males $=$ 49; females $=47$ ) of which 111 participants performed the attention task before the start of treatment of ChEIs (Reminyl and Exelon). Medications included 3 different maximum doses of Reminyl (8/16/24) and 2 of Exelon (4.6/9.5). Patients were treated by a dose chosen by the geriatricians, which was adapted during the treatment period which makes it hard to account for. Neither plasma levels nor compliance was measured. Residual analyses revealed that 3 (2 AD; $1 \mathrm{VaD})$ of
111 participants $(\mathrm{AD}=88, \mathrm{VaD}=23)$ had a mean $\mathrm{RT}$ score which was 3 or more standard deviations (SD) from the mean. This finding in combination with the observations of the test leader suggested that the patients might not have understood the instructions, and therefore these subjects were excluded from the analyses.

Participants had a diagnosis of $\mathrm{AD}$ or $\mathrm{VaD}$ in line with the DSM-IV criteria. They were recruited in the Albert Schweitzer Hospital in Dordrecht, the Netherlands. Clinical diagnoses were made by a senior geriatrician. Patients with delirium or patients who were immobile did not participate in this study. All participants gave written informed consent after having been informed about the purpose of the study research. The study has approval of the medical ethical committee of the Albert Schweitzer Hospital. In this study, an attention task was administered to measure sustained attention before medication was given. In addition, the MiniMental State Examination (MMSE) and a short composed questionnaire measuring adverse side effects of the treatment with ChEIs (Appendix B) were administered.

2.1. Attention Task. Participants were asked to press a button when one of a total of 30 stimuli was presented on the screen. The time it took for the participant to react to each stimulus and the summation of the total RT was registered in milliseconds. The task took less than two minutes. The first 5 trials were considered as practice tasks and therefore not included in the analyses. Requirements of participating were that the patient could be seated behind a desk and was able to understand the instructions. Before performing the attention task the participant was verbally instructed to react whenever the white figure would turn into orange. As soon as the color turned orange the timer started measuring the time until the participant reacted to the stimulus in milliseconds.

2.2. Mini-Mental State Examination (MMSE). The MiniMental State Examination (Appendix A) is the most commonly used screening test for the evaluation of cognitive impairment. During this examination orientation, recall, attention, recording capacity, language, and constructive praxis were tested [14]. The MMSE can be used to estimate the severity of dementia, whether there is no dementia present (24-30), mild dementia (20-23), moderate (10-19), or a severe case of dementia (0-9) [15].

2.3. Questionnaire. The questionnaire (Appendix B) consisted of six dichotomous questions (yes, no), one question with three options (none, doubt, and good) and two open questions about the medication and the side effects. Side effects were measured through questioning by geriatricians of any additional side effects since the start of medication (yes/no). In addition, geriatricians reported what other side effects there were, not the severity. Examples of side effects are, but not limited to, dizziness, nausea, and agitation.

2.4. Statistical Analyses. The program SPSS version 22 was used for the statistical analyses. The study had a quasiexperimental design, with two patient groups ( $\mathrm{AD}$ and $\mathrm{VaD}$ ) 
TABle 1: Demographic data and comparisons between groups on the task and questionnaire.

\begin{tabular}{lccccc}
\hline & AD & VaD & df & $F$-value & $p$-value \\
\hline Age (years) & $77.93 \pm 6.552$ & $78.93 \pm 7.8$ & 70 & .242 & .624 \\
Gender M : F & $27: 30$ & $9: 5$ & 70 & 7.274 & .388 \\
MMSE & $20.54 \pm 3.928$ & $21.29 \pm 4.250$ & 70 & .63 \\
Number of side effects & $.656 \pm .701$ & $.600 \pm .894$ & 63 & 3.023 \\
Occurrence of side effects & $25: 27$ & $4: 8$ & 63 & .840 & .087 \\
Mean RT (ms) & $829.840 \pm 445.751$ & $1099.162 \pm 519.219$ & 70 & 3.845 & .363 \\
\hline
\end{tabular}

Mean \pm SD, MMSE $=$ Mini-Mental Stage Examination, $\mathrm{RT}=$ reaction time, $\mathrm{AD}=$ Alzheimer's disease, $\mathrm{VaD}=$ vascular dementia, and $\mathrm{df}=\mathrm{degrees}$ of freedom

who completed the attention task, MMSE, and questionnaire. An independent $t$-test was used to test a difference between the mean RT of both patient groups, controlling for unequal variances. One-way analyses of variance (ANOVAs) were performed to test the differences in age, gender, MMSE, number, and occurrence of side effects, between the patient groups. Subsequent ANOVAs and regression analyses were used to evaluate if RT differed between the side effect groups, for the total sample and separate patient groups. Furthermore, an additional regression analysis was used to test for a difference in RT between patients with and without side effects, for the total group and separate patient groups. In addition, Pearson correlation between MMSE score and RT was calculated using "Bivariate correlation," for the total group as well as separately for $\mathrm{AD}$ and $\mathrm{VaD}$.

\section{Results}

3.1. Differences between $A D$ and $V a D$. In this study data from 71 of the 111 participants were included in the analyses. These were the participants who fulfilled the requirements of (1) performing the attention task before taking Exelon or Reminyl, (2) scoring above MMSE score 12, and (3) having a mean RT not deviating more than 3 SD from the mean. The first 5 trials were considered to be practice trials. Therefore, mean RT was calculated by averaging all trials excluding the first 5 trials.

Patient groups tended to differ on the number of side effects (Table 1) as AD patients would experience more side effects than VaD patients. Patient groups did not significantly differ on age, gender, MMSE score, or occurrence of side effects (yes/no). An independent $t$-test revealed a significant difference between patient groups on mean RT, when only trial scores were included that deviated less than 3 SD from the mean $(p$ [1-tailed] $=.027, p$ variances unequal [1-tailed $]=$ .045); as predicted $\mathrm{VaD}$ patients had a longer RT than AD patients (Figure 1).

3.2. Relation between the Number of Side Effects and RT. To test the relation between the number of side effects and RT, a regression analysis was performed, controlling for age, gender, MMSE score, and diagnosis. We excluded data from subjects when mean RT scores were more than 3 SDs from the sample mean. The correlation between mean RT and number
TABLE 2: Results ANOVA mean RT by side effects.

\begin{tabular}{lccc}
\hline Sample & IV & $F$ & $p$ \\
\hline Total group & Number of side effects & .423 & .737 \\
Total group & Occurrence of side effects & .000 & .998 \\
$\mathrm{AD}$ & Number of side effects & .471 & .704 \\
$\mathrm{VaD}$ & Number of side effects & 1.33 & .331 \\
$\mathrm{AD}$ & Occurrence of side effects & .023 & .881 \\
$\mathrm{VaD}$ & Occurrence of side effects & .319 & .585 \\
\hline
\end{tabular}

$\mathrm{IV}=$ independent variable, $\mathrm{RT}=$ reaction time, $\mathrm{AD}=$ Alzheimer's disease $\mathrm{VaD}=$ vascular dementia, $F=F$ value, and $p=p$ value.

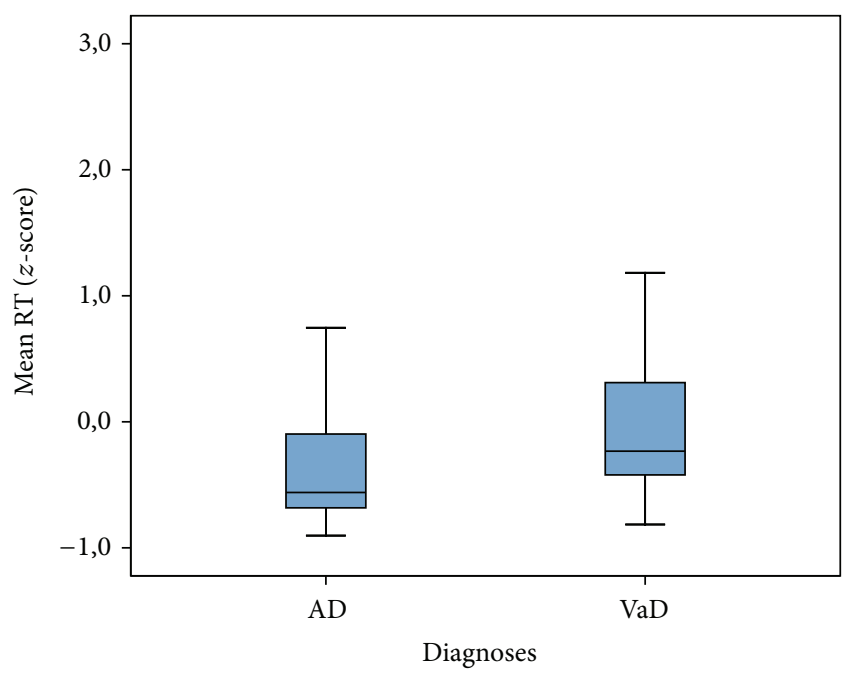

Figure 1: Mean RT ( $z$-score) and SD bars for $\mathrm{AD}$ and VaD patients.

of side effects was not significant $(r=-.041, p$ [1-tailed $]=$ .188) when calculated for the total group of subjects. In contrast, (bivariate) correlation analyses performed separately in each patient group revealed a different direction of the correlations between the mean RT and the number of side effects; namely non-significant for the AD $(r=.048, p[1-$ tailed $]=.37)$ versus significant for the $\operatorname{VaD}$ group $(r=-.445$, $p[1$-tailed $]=.037)$.

A one-way ANOVA with "number of side effects" as independent variable in the total group showed that "number of side effects" had no effect on the mean RT (Tables 2 and 3 ). Another ANOVA was performed, using the grouped variable 


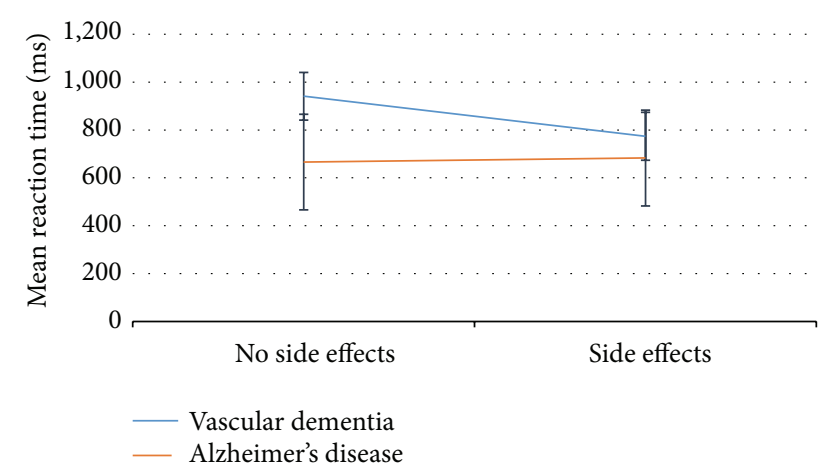

FIGURE 2: Occurrence of side effects versus mean RT trial (ms), for $\mathrm{AD}$ and $\mathrm{VaD}$.

TABLE 3: Number of side effects with associated mean RT.

\begin{tabular}{lcccccc}
\hline & $\begin{array}{c}\text { Number of } \\
\text { side effects }\end{array}$ & Total & AD & $n$ & VaD & $n$ \\
\hline & 0 & 682.914 & 666.391 & 25 & 941.340 & 4 \\
Mean RT & 1 & 809.126 & 632.898 & 16 & 1156.973 & 3 \\
$(\mathrm{~ms})$ & 2 & 723.281 & 794.075 & 10 & 599.493 & 3 \\
& 3 & 441.867 & 396.320 & 1 & 464.640 & 2 \\
\hline
\end{tabular}

$\mathrm{RT}=$ reaction time, $\mathrm{AD}=$ Alzheimer's disease, $\mathrm{VaD}=$ vascular dementia, and $n=$ number of participants.

TABLE 4: Occurrence of side effects with the accompanied mean RT.

\begin{tabular}{lcccccc}
\hline & $\begin{array}{c}\text { Occurrence } \\
\text { of side effects }\end{array}$ & Total & AD & $n$ & VaD & $n$ \\
\hline $\begin{array}{l}\text { Mean RT } \\
(\mathrm{ms})\end{array}$ & No & 704.3149 & 666.3909 & 25 & 941.3400 & 4 \\
\hline
\end{tabular}

$\mathrm{RT}=$ reaction time, $\mathrm{AD}=$ Alzheimer's disease, $\mathrm{VaD}=$ vascular dementia, and $n=$ number of participants.

"occurrence of side effects (yes/no)" instead of the "number of side effects", which also showed no effect on the mean RT (Tables 2 and 4). Splitting up the total group in $\mathrm{VaD}$ and $\mathrm{AD}$ groups to find an effect for the number of side effects on the mean RT, did not lead to significant results either (Table 2). These results are probably due to the magnitude of the SD; see Figure 2.

In summary, despite the nonsignificant results of the ANOVA, a significant negative correlation was observed for the $\mathrm{VaD}$ group between the number of side effects and the mean RT. This correlation indicated a longer RT when a patient did not or experienced less side effects compared to when he/she experienced more side effects (Table 3). There appeared to be no such trend for the number of side effects and RT for the total group as well as for the AD group; see Table 3.

3.3. Relation MMSE Score and RT. Correlation analyses calculated for the total sample showed a nonsignificant correlation between RT and the MMSE score, $r=-.172$, $p=.151$ (Table 5). Splitting the groups resulted in different findings. For the AD group, the MMSE did not correlate with
TABLE 5: Overview of correlation analyses with MMSE score and mean RT.

\begin{tabular}{lcc}
\hline Group & Statistics & Mean RT 6-30 \\
\hline \multirow{2}{*}{ Total } & $r$ & -.172 \\
& $p$ & .151 \\
\hline \multirow{2}{*}{$\mathrm{AD}$} & $r$ & -.035 \\
& $p$ & .796 \\
\hline \multirow{2}{*}{$\mathrm{VaD}$} & $r$ & -.738 \\
& $p$ & $\mathbf{. 0 0 3}$ \\
\hline
\end{tabular}

$\mathrm{RT}=$ reaction time, $\mathrm{AD}=$ Alzheimer's disease, $\mathrm{VaD}=$ vascular dementia, $r=$ correlation, and $p=p$ value.

the mean RT of the sustained attention task as $r$ did not suggest a strong positive or negative correlation, $r=-.035$, $p=.796$, (Table 5). For the VaD group, the MMSE negatively correlated with the mean RT $(r=-.738, p=.003)$ (Table 5). In addition, the value of $r$ indicates a strong negative correlation between MMSE and RT, a longer RT when the patient had a lower (worse) score on the MMSE.

\section{Conclusions}

4.1. Hypotheses. The aim of this study was to test the hypothesis of Van Gool in order to predict the side effects of ChEIs in $\mathrm{AD}$ and $\mathrm{VaD}$ patients. The hypothesis stated that patients with dementia with cholinergic deficit symptoms will benefit from ChEI medication whereas patients without these symptoms will suffer from side effects because of overstimulation of the cholinergic system. As patients with cholinergic deficit symptoms have attention problems, they will have a longer $\mathrm{RT}$, leading to the following 3 hypotheses: (1) AD patients with a longer RT than average on the attention task will benefit more from treatment than patients with an average or faster one and thus should show less side effects, (2) $\mathrm{VaD}$ patients with a longer $\mathrm{RT}$ than average on the attention task will benefit more from treatment than patients with an average or faster one and thus should show fewer side effects, and (3) as the task measures sustained attention which is impaired at an early stage of $\mathrm{VaD}$ but usually preserved in $\mathrm{AD}$, it is predicted that $\mathrm{VaD}$ patients will have a longer $\mathrm{RT}$ on the attention task than AD patients. Hypothesis 1 can be rejected as correlation analyses revealed no specific direction or significant correlation between the mean RT and the number of side effects for $\mathrm{AD}$ patients, as well as for the mean RT and the occurrence of side effects for the $\mathrm{AD}$ group. Furthermore, a one-way ANOVA confirmed these results. Hypothesis 2 was confirmed. Correlation analyses indicated a negative correlation between the mean RT and the number of side effects for the VaD group. Nevertheless, ANOVA results are nonsignificant, which is probably due to the magnitude of the SD of the RT scores. Hypothesis 3 was also confirmed using the mean $\mathrm{RT}$ that is corrected for deviations of 3 or more SD from the mean, $p<.027$, which is in line with the previous research.

4.2. MMSE and RT. In addition, to validate the attention task a correlation analysis was performed between RT and 
TABLE 6: Mini-Mental State Examination (MMSE).

\begin{tabular}{lc}
\hline Item MMSE & Function \\
\hline M_time & Orientation \\
M_place & Direct reproduction \\
M_reproduction & Attention \\
M_attention & Memory \\
M_memory & \\
M_language & \\
M_repeat & Language \\
M_followinginstructions & \\
M_closeeyes & \\
M_writingsentice & \\
M_figure & Construction \\
\hline
\end{tabular}

the MMSE score, resulting in nonsignificant results. Splitting up the sample in $\mathrm{AD}$ and $\mathrm{VaD}$ groups showed that although the MMSE score did not correlate with the mean RT for the $\mathrm{AD}$ group, it did correlate negatively for the $\mathrm{VaD}$ group. In other words, a lower (worse) MMSE score was related to a longer RT for the VaD group. This result might be explained by the notion that $\mathrm{VaD}$ is associated with white matter loss and more severe periventricular hyperintensities [12], which are related to slowing of mental processing speed, as well as other deficits in cognition [13]. These lead to a longer RT as well as to more general cognitive problems compared with $\mathrm{AD}$ patients. This is in line with the trend that $\mathrm{VaD}$ patients experience more side effects than $\mathrm{AD}$ patients.

4.3. Summary. Thus, the attention task tends to predict the side effects in $\mathrm{VaD}$, but not in the $\mathrm{AD}$ group. Perhaps the task is only a valid method to predict side effects in patients with $\mathrm{VaD}$ and not in $\mathrm{AD}$ as it might not be subtle enough to measure the cholinergic deficit symptoms in patients without general cognitive deficits. However, patient groups were not equal in this study, and medication doses varied, which might have undermined the effect for $\mathrm{AD}$. More research is needed to predict the side effects in both $\mathrm{VaD}$ and $\mathrm{AD}$ patients.

\section{Appendices}

\section{A.}

See Table 6.

\section{B.}

See Table 7.

\section{Conflict of Interests}

The authors declare that there is no conflict of interests regarding the publication of this paper.
TABLE 7: Questionnaire about side effects.

\begin{tabular}{lc}
\hline Item & Response \\
\hline Medication & Open question \\
Dyspnea & Yes/no \\
Nausea & Yes/no \\
Diarrhea & Yes/no \\
Reduce appetite & Yes/no \\
Rash plaster & Yes/no \\
Other side effects & Yes/no \\
What other side effects & Open question \\
Satisfaction medication & Negative/doubt/positive \\
\hline
\end{tabular}

\section{Acknowledgments}

The authors are grateful to Dr. Jan B. Deijen and Mr. Matthieu $\mathrm{H}$. Beaumont for critically reading the paper.

\section{References}

[1] American Psychiatric Association, Diagnostic and Statistical Manual of Mental Disorders, American Psychiatric Association, Washington, DC, USA, 4th edition, 2000.

[2] V. T. Griffith, "Diagnose and treat mild to moderate Alzheimer's disease," The Nurse Practitioner, vol. 27, no. 12, pp. 13-25, 2002.

[3] M. Grothe, L. Zaborszky, M. Atienza et al., "Reduction of basal forebrain cholinergic system parallels cognitive impairment in patients at high risk of developing Alzheimer's disease," Cerebral Cortex, vol. 20, no. 7, pp. 1685-1695, 2010.

[4] T. Erkinjuntti, G. Román, S. Gauthier, H. Feldman, and K. Rockwood, "Emerging therapies for vascular dementia and vascular cognitive impairment," Stroke, vol. 35, no. 4, pp. 1010$1017,2004$.

[5] A. W. Lemstra, P. Eikelenboom, and W. A. van Gool, "The cholinergic deficiency syndrome and its therapeutic implications," Gerontology, vol. 49, no. 1, pp. 55-60, 2003.

[6] G. Pepeu, M. G. Giovannini, and L. Bracco, "Effect of cholinesterase inhibitors on attention," Chemico-Biological Interactions, vol. 203, no. 1, pp. 361-364, 2013.

[7] D. M. Malone and J. Lindesay, "Cholinesterase inhibitors and cardiovascular disease: a survey of old age psychiatrists' practice," Age and Ageing, vol. 36, no. 3, pp. 331-333, 2007.

[8] H. Kavirajan and L. S. Schneider, "Efficacy and adverse effects of cholinesterase inhibitors and memantine in vascular dementia: a meta-analysis of randomised controlled trials," The Lancet Neurology, vol. 6, no. 9, pp. 782-792, 2007.

[9] S. S. Gill, G. M. Anderson, H. D. Fischer et al., "Syncope and its consequences in patients with dementia receiving cholinesterase inhibitors: a population-based cohort study," Archives of Internal Medicine, vol. 169, no. 9, pp. 867-873, 2009.

[10] N. L. Graham, T. Emery, and J. R. Hodges, "Distinctive cognitive profiles in Alzheimer's disease and subcortical vascular dementia," Journal of Neurology, Neurosurgery \& Psychiatry, vol. 75, no. 1, pp. 61-71, 2004.

[11] E. Scherder, Aging and Dementia. Neuropsychology, Motor Skills and Pain, VU University Press, Amsterdam, The Netherlands, 2011.

[12] R. Barber, P. Scheltens, A. Gholkar et al., "White matter lesions on magnetic resonance imaging in dementia with Lewy bodies, 
Alzheimer's disease, vascular dementia, and normal aging," Journal of Neurology Neurosurgery and Psychiatry, vol. 67, no. 1, pp. 66-72, 1999.

[13] H. C. Chui, "Subcortical ischemic vascular dementia," Neurologic Clinics, vol. 25, no. 3, pp. 717-740, 2007.

[14] S. Bagnoli, Y. Failli, I. Piaceri et al., "Suitability of neuropsychological tests in patients with vascular dementia (VaD)," Journal of the Neurological Sciences, vol. 322, no. 1-2, pp. 41-45, 2012.

[15] A. Dalvi, "Alzheimer's disease," Disease-a-Month, vol. 58, no. 12, pp. 666-677, 2012. 


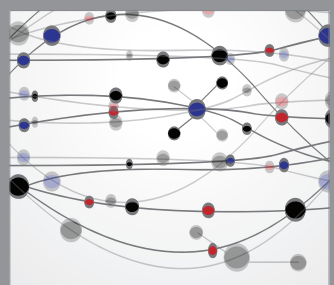

The Scientific World Journal
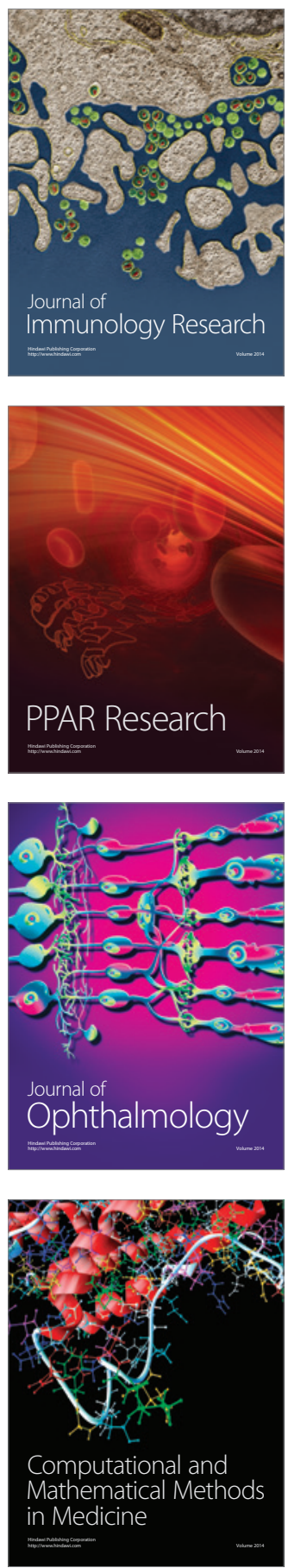

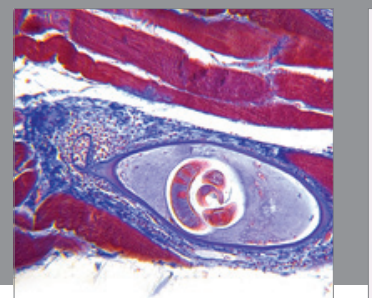

Gastroenterology

Research and Practice
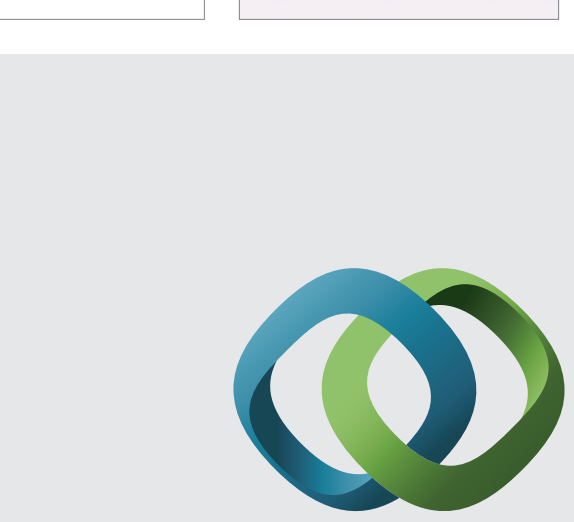

\section{Hindawi}

Submit your manuscripts at

http://www.hindawi.com
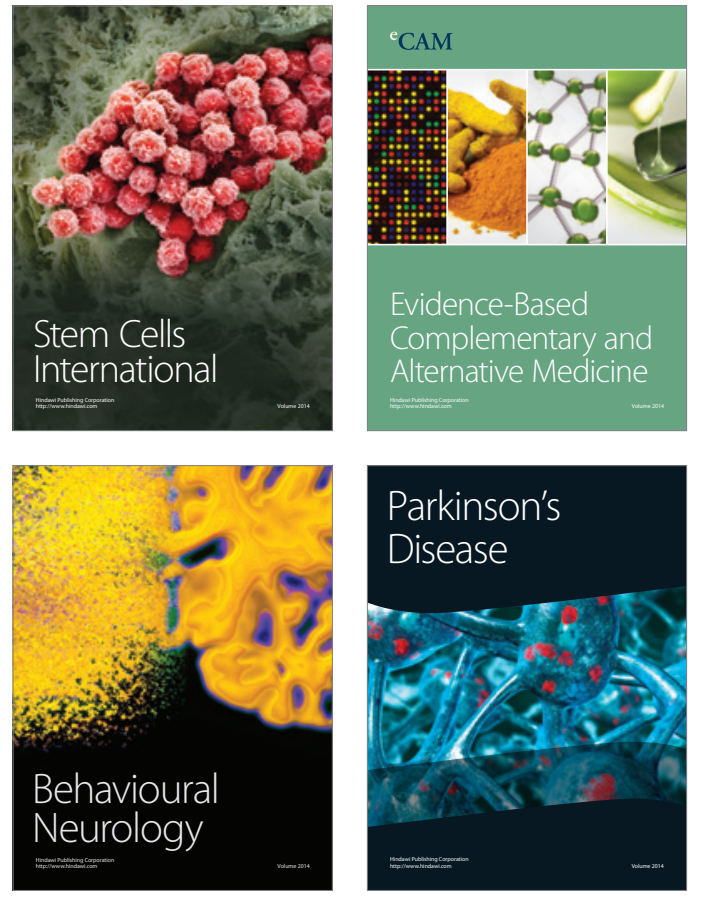
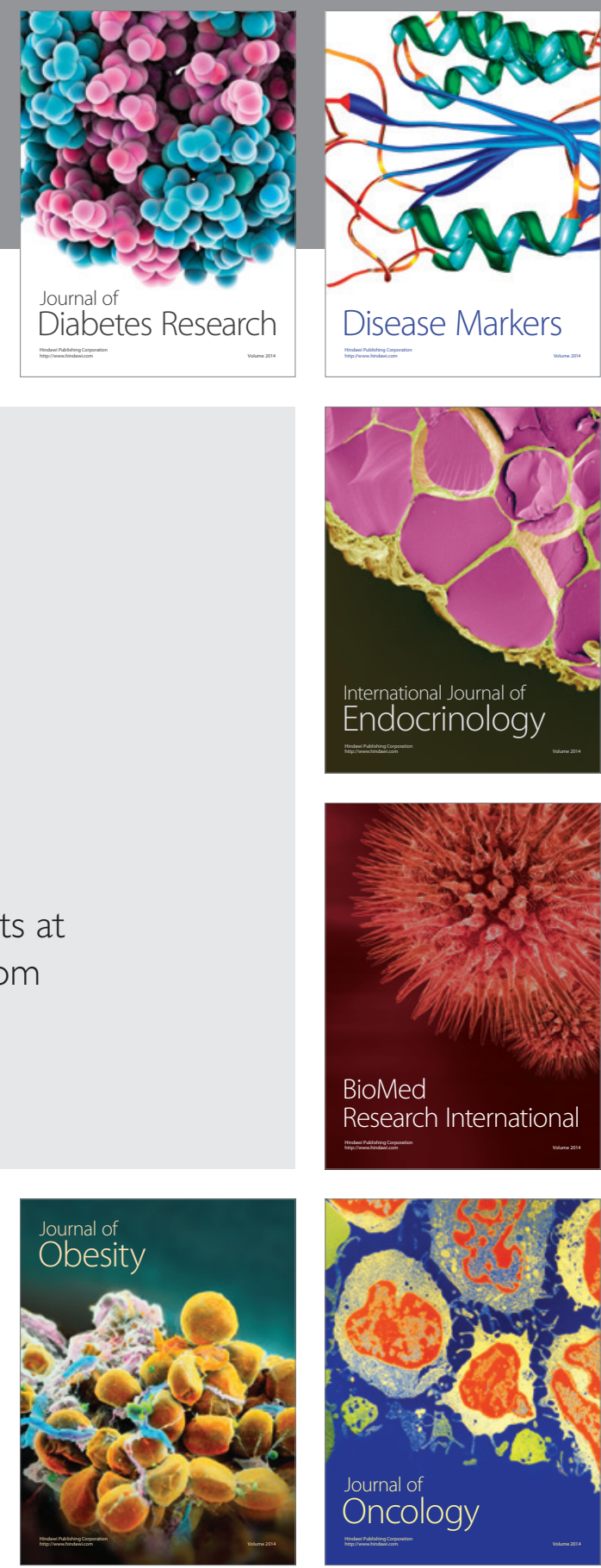

Disease Markers
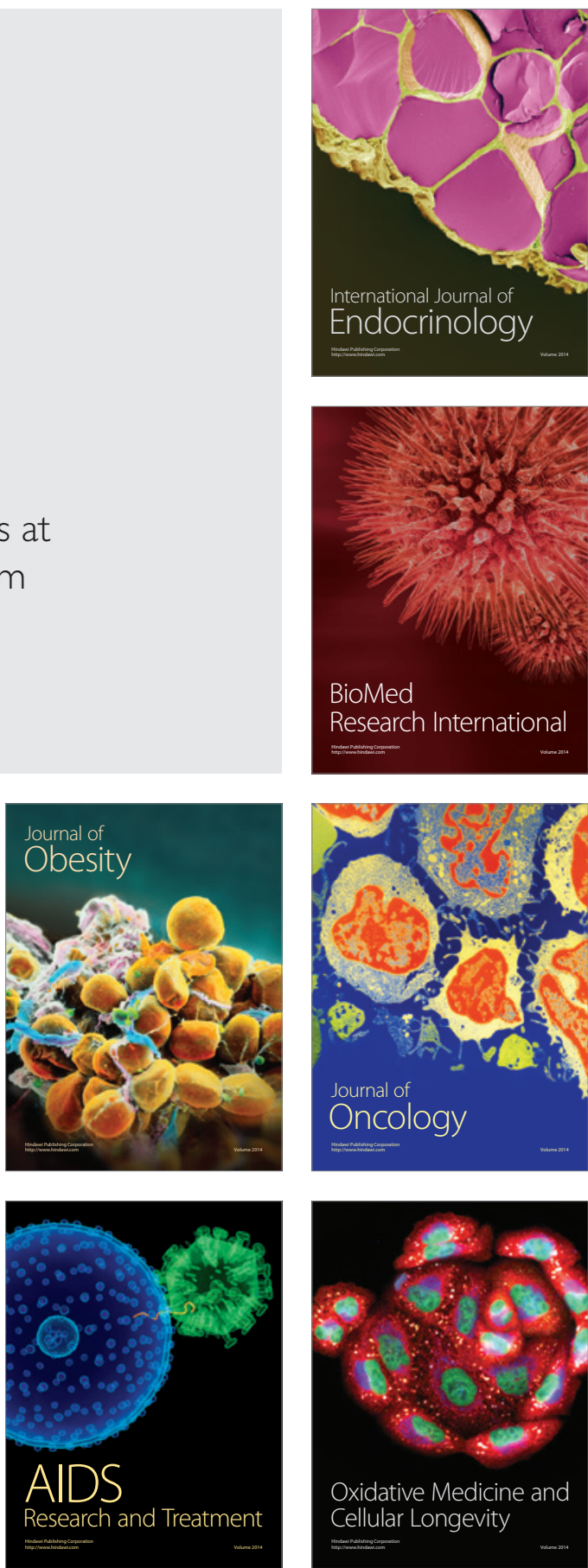